\title{
BI-ANGULAR LENS FOR MATERIAL CHARACTERIZATION
}

\author{
G.G. Yaralıg̃lu, A. Atalar, H. Köymen \\ Electrical and Electronics Engineering Department \\ Bilkent University, 06533 Bilkent, Ankara, TURKEY
}

\begin{abstract}
In this paper a new lens design is pro. posed for characterization of layered materials. Lamb wave lens employs Lamb waves for this purpose since these waves propagate along interfaces. However, below cut-off angle, the critical angles of Lamb wave modes are low and the generated $V(z)$ curves have small num. ber of oscillations, which in turn causes measurement difficulties and accuracy degradation. Bi-angular lens described in this paper, generates an extra obliquely incident wave, instead of normally incident beam, in order to provide the reference specular reflection. Simulation results as well as experimental results are presented and it is shown that a high sensitivity can be obtained by using this new lens.
\end{abstract}

\section{Introduction}

It is well-known that when a solid half space, coated by a layer of material with shear velocity less than the substrate's shear velocity, is insonified by an acoustic beam, there exists many leaky Lamb wave modes [1][3]. These modes are observed as oscillations in V(z) curves and their velocities can be calculated by spectral analysis or model based algorithms [4].

The accuracy of the Leaky wave velocity extraction methods depends on the number of oscillations in the $\mathrm{V}(\mathrm{z})$ curve. The period of the oscillations is given by the formula:

$$
\Delta z=\frac{\lambda_{0}}{2\left(1-\cos \theta_{M}\right)},
$$

where $\lambda_{0}$ and $\theta_{M}$ are the wavelength in the water and the mode critical angle, respectively [5]. Mode velocity is calculated by:

$$
v_{M}=\frac{v_{0}}{\sin \theta_{M}}
$$

and $v_{0}$ is the sound velocity in the water. The period of the oscillations increase with the mode velocity. Hence modes that exist around longitudinal critical angles give rise to small number of oscillations. This limitation causes measurement problems and degrades the accuracy of velocity extraction. Interference pattern in the $V(z)$ curve obtained from conventional lenses or Lamb wave lenses are due to the interference between the nonspecular reflection and the normally incident beam. One way to increase the difference between the angles of the specular and the nonspecular reflected beams, and hence to increase the number of oscillations in $V(z)$, is to employ another obliquely incident beam as the reference specular component instead of the normal beam. The incidence angle of the specular beam is chosen to maintain an angular difference, with the incidence angle of leaky component, such that best accuracy in characterization is achieved.

The proposed bi-angular lens contains two conical surfaces such that the vortex angle of the first surface is dictated by the incidence angle of the Lamb wave mode, where as the vortex angle of the second mode is selected to provide a specular reflection at an appropriate incidence angle. The central part is blocked by an acoustically opaque material. In the following section, the geometry of the lens is described and design considerations are discussed. Then, the response of the lens as a function of defocus distance is investigated and compared with the Lamb wave lens. Theoretical simulations are done to deduce the performance of the lens. Experimental results are also presented and compared with the theory.

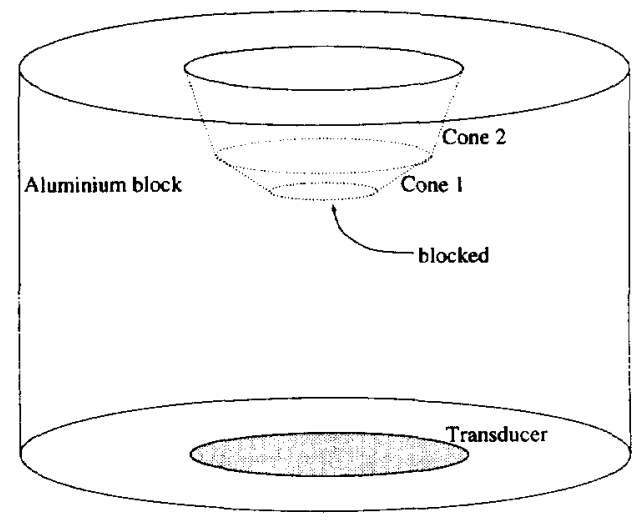

Figure 1: Bi-angular Lens

\section{Bi-angular Lens}

Bi-angular lens is composed of two conical surfaces as depicted in Figure 1. Sound waves are first refracted by the inclined surfaces before they reach to the object 
surface. Central part of the recess is blocked. Hence, material surface is insonified by two obliquely incident beams. The inclination angle of the inner surface is set such that incidence angle of the refracted beam at this conical surface matches with one of the mode critical angles. This beam generates a leaky Lamb wave mode on material surface. The incidence angle of the specular beam is higher than the Rayleigh angle of the layer, hence the reflected field from the material surface contains only specular component. Interference pattern of the specular beam and the leaky field is observed as oscillations in $\mathrm{V}(\mathrm{z})$ curve.

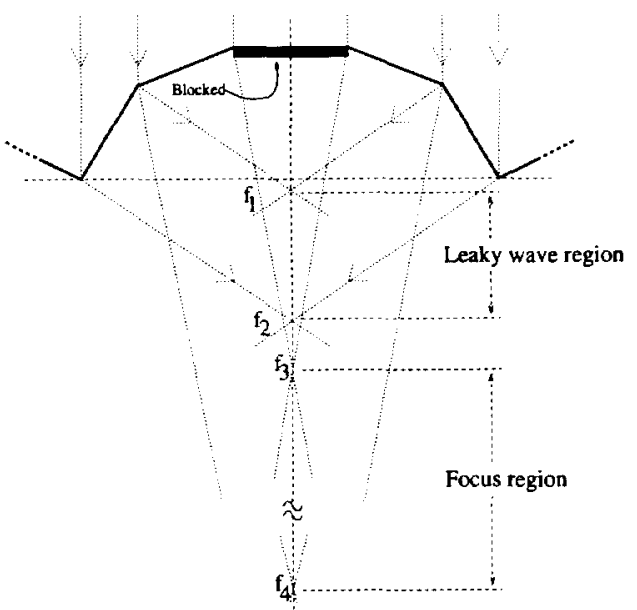

Figure 2: Bi-angular Lens Geometry

In Figure 2, the traces of the acoustic rays refracted at the conical surfaces are shown. If $z$ is between $f_{1}$ and $f_{2}$, the leaky wave region, oscillations in $\mathrm{V}(\mathrm{z})$ curve is due to the interference of the leaky field and the specular beam. Maximum signal amplitude is reached at the mid-point of this region. Between $f_{3}$ and $f_{4}$, the focus region, lens can collect only specularly reflected beam refracted at inner cone (Figure 4). Distance between $f_{1}$ and $f_{2}$ is maximized to get more oscillations in $V(z)$ curves and $f_{1}$ is placed near the aperture. To obtain reference curve, materials whose critical angles does not match with the incidence angle of the inner cone can be used. In this case, although there is no leaky wave excitation, there exist small oscillations in leaky wave region due to the diffraction. To minimize diffraction, radius of the inner cone, $r_{2}$, and the lens aperture, $r_{3}$, are chosen to prevent interference of the specularly reflected beams. In other words, $f_{2}$ is placed before $f_{3}$.

\section{Bi-angular Lens v.s. Lamb Wave Lens}

Bi-angular lens is an extension to the Lamb wave lens [6] and it is especially useful for material characterization by using high velocity modes. In Figure 3, calculated reflection coefficient for 600 micro-meter thick copper layer on steel substrate is shown. There exists a leaky mode around the longitudinal critical angle of steel. Frequency is chosen as $24.6 \mathrm{MHz}$. The leaky Lamb wave mode is observed as a sharp phase transition at $15.50^{\circ}$, and the corresponding mode velocity is 5646 meter/sec. This mode leaks both back to the coupling medium and into the steel substrate. This phenomenon is observed as a dip in the reflection coefficient. Reflection coefficient calculation is performed by employing boundary matrix method [7].

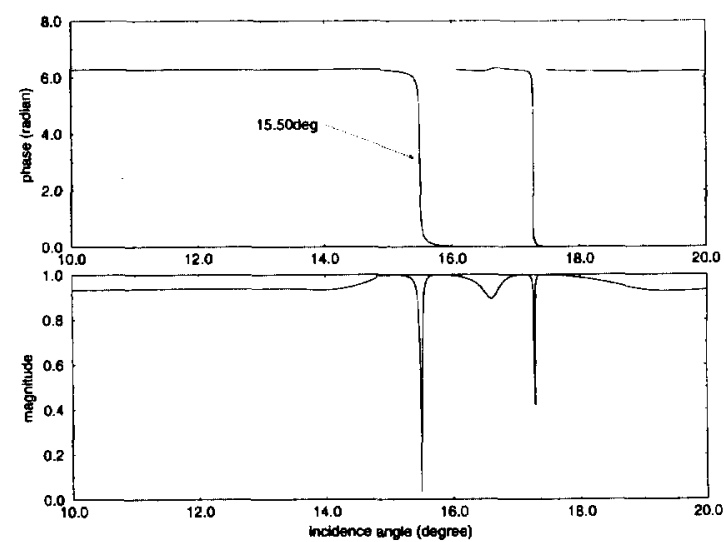

Figure 3: Calculated reflection coefficient of 600 micrometer thick copper layer on steel substrate at $24.6 \mathrm{MHz}$.

In Figure 4, simulated $V(z)$ curves obtained from Lamb wave lens and bi-angular lens are shown. The lens dimensions are provided in the figure. The wedge angle of the inner and the outer cones are chosen as $20.0^{\circ}$ and $70.0^{\circ}$, respectively. Corresponding incidence angles are $15.40^{\circ}$ and $57.24^{\circ}$. For the beam refracted at the inner cone, most of the power converted to the leaky Lamb wave mode. In order to obtain a V(z) from Lamb wave lens, the planar central part must be kept open. For bi-angular lens, on the other hand it must be blocked. In Figure 4, V(z) for both lenses are given. The amplitudes of the oscillations are nearly the same in leaky wave region, but the number of oscillations increases significantly, for the case of bi-angular lens. The period of the oscillations are calculated by using the formula:

$$
\Delta z=\frac{\lambda_{0}}{2\left(\cos \theta_{M}-\cos \theta_{I}\right)},
$$

where $\theta_{I}$ is the incidence angle of the outer cone.

\begin{tabular}{|l|r|r|r|}
\hline Material & $v_{l}(\mathrm{~m} / \mathrm{sec})$ & $v_{s}(\mathrm{~m} / \mathrm{sec})$ & $\rho\left(\mathrm{kg} / \mathrm{m}^{3}\right)$ \\
\hline Water & 1508.9 & - & 1000.0 \\
Copper & 5010.0 & 2290.0 & 8933.0 \\
Steel & 5900.0 & 3200.0 & 7900.0 \\
\hline
\end{tabular}

Table 1: Material parameters used in simulations. 


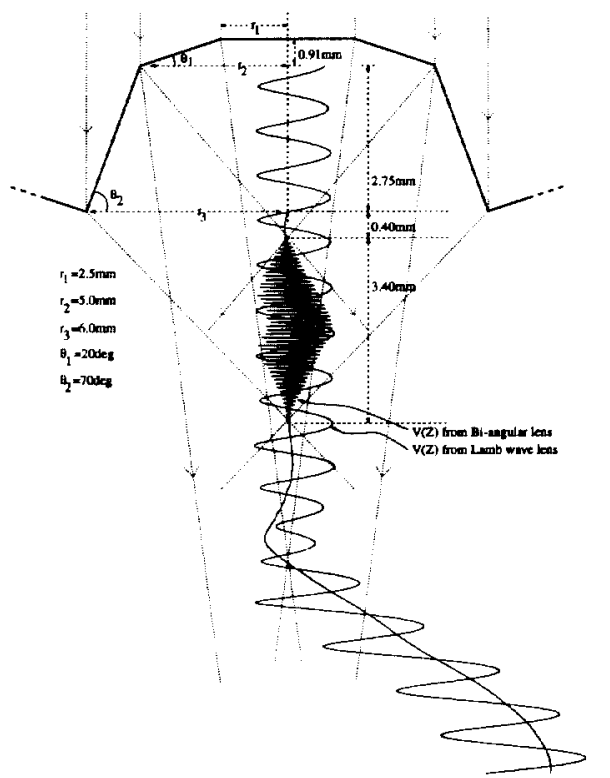

Figure 4: V(z) curves obtained by using Lamb wave lens and $\mathrm{Bi}$-angular lens

Simulation program, that calculates $V(z)$, is written by employing both angular spectrum methods and ray theory. The potential distribution at the transducer is propagated through the lens material by taking the Fourier transform of the field and adding phase terms. Due to the symmetry it is possible to use one dimensional Quasi Fast Hankel Transform to calculate two dimensional Fourier transform. After finding the potential distribution at the back of the recess, distribution at the aperture is calculated by using ray theory. The distribution at the aperture is shown in Figure 5 for the bi-angular lens used in simulation. Ray theory analysis does not consider beam divergence due to the diffraction. Hence, beam widths depicted in figure are less than actual beam widths.

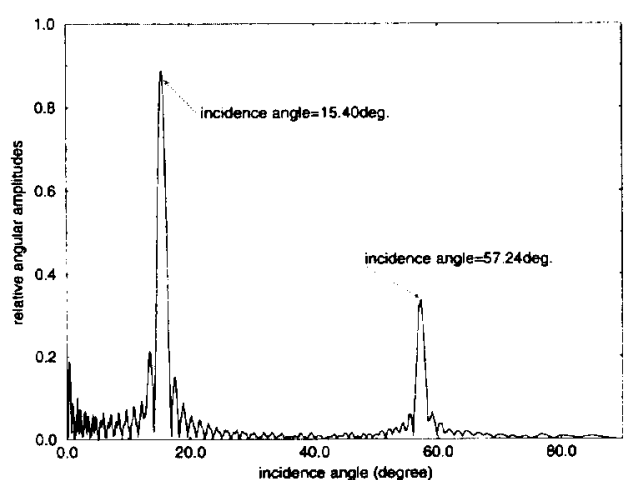

Figure 5: Angular distribution at the aperture of biangular lens

\section{Experiments}

In experiments, the lens whose dimensions are shown in Figure 4 is used. Center part is blocked by using polystyrene. The lens material is aluminum whose longitudinal velocity is 6420 meter/sec. The alignment of the lens and the object is achieved by maximizing the transducer output voltage when $z$ is between $f_{3}$ and $f_{4}$ (Figure 2), since the maximum signal level is reached when the central part is parallel to the material surface.

The material under observation is 600 micro-meter thick copper layer on steel substrate. Copper is plated on steel electrochemically.

Z-scan is achieved by using a computer controlled motor with $0.1 \mu$ meter accuracy and $V(z)$ is taken at 1000 points over a defocus range of $4 \mathrm{~mm}$.

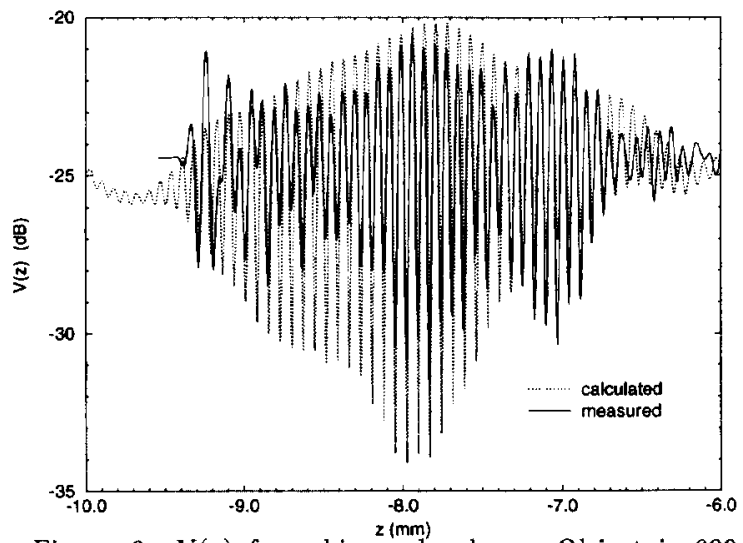

Figure 6: $V(z)$ from bi-angular lens. Object is 600 micro-meter thick copper layer on steel substrate

The experimental data depicted in Figure 6 is filtered by a band pass filter whose cut-off angles are at $5^{\circ}$ and $50^{\circ}$ in angular domain. Base-line variations of $\mathrm{V}(\mathrm{z})$ curve are due to the background pulses emerge from reflected shear echoes and high frequency components are due to the imperfection of switches. These oscillations are eliminated by band pass filtering.

In Figure 7, $\mathrm{V}(\mathrm{z})$ curves obtained from Lamb wave lens, bi-angular lens and spherical lens are shown. The apertures of the spherical and the bi-angular lens are the same. The cone angle of the spherical lens is chosen as $17^{\circ}$. Hence, only the mode shown in Figure 3 is excited. The period of the oscillations from Lamb wave lens and spherical lens are the same, whereas for the bi-angular lens it is small. The amplitudes of the oscillations for Lamb wave lens and the bi-angular lens are comparable and both are an order of magnitude higher than that of the spherical lens.

\section{Conclusion}

$V(z)$ technique for characterization of materials with high velocity modes suffers from the fact that variation 


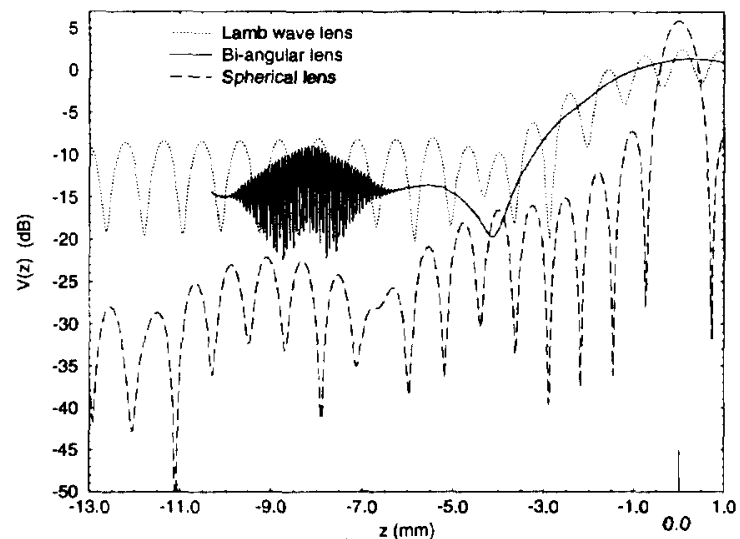

Figure 7: Simulated V(z) curves. Material under observation is 600 micro-meter thick copper on steel substrate and frequency $=24.6 \mathrm{MHz}$.

of $V(z)$ with respect to $z$ is slow and hence the accuracy of velocity extraction process is degraded. Proposed lens geometry overcomes this difficulty. $V(z)$ obtained with bi-angular lens provides a large number of oscillations in leaky wave region. Its leaky wave excitation efficiency is the same as the Lamb wave lens. Hence, the modulation index in the $V(z)$ curves maintains a high signal-to-noise ratio.

The accuracies of the spectral or model based velocity extraction algorithms depend on the number of oscillations in $V(z)$ curves. $V(z)$ 's from Lamb wave and bi-angular lenses contain two peaks in angular domain. These peaks correspond to the base line variations which we called spectral peak, and oscillations due to the leaky waves known as leaky wave peak. In the case of Lamb wave lens, for high velocity modes, these peaks are close to each other and spectral peak affects the leaky wave peak, resulting in the mis-calculation of the place of the maximum value of the leaky wave peak. For bi-angular lens, since the frequency of oscillations is increased, in spectral domain these peaks stay apart from each other and baseline variations do not effect the leaky wave peak.

The measurement accuracy of the bi-angular lens depends on the match between the incidence angle and the leaky wave critical angle, as in the case of Lamb wave lens. In velocity measurements, the absolute accuracy of Lamb wave lens is $1 \%$ [4] as long as the critical angle stays within in the angular width of the incident beam. For bi-angular lens, by a proper windowing on $\mathrm{V}(\mathrm{z})$ curve, an absolute accuracy of $0.5 \%$ can be achieved.

The proposed geometry is not suitable for the anisotropy measurements, since it has not direction sensitivity. A version of $V$-groove lens [4] with the same cross section of the bi-angular lens shown in Figure 2 can be constructed for anisotropy measurements.

\section{References}

[1] Nayfeh, A. H., Chimenti, D. E., "Reflection of finite acoustic beams from loaded and stiffened halfspaces", J. Acous. Soc. Am., Vol. 75, pp. 1360$1368,1984$.

[2] Chimenti, D. E., Nayfeh, A. H., "Leaky Lamb waves in fibrous composite laminates",J. Appl. Phys., Vol. 58, pp. 4531-4538.

[3] Chimenti, D. E., Nayfeh, D. L., Butler, D. L., "Leaky Rayleigh waves on a layered halfspace", $J$. Appl. Phys., Vol. 53, pp. 170-176, 1982.

[4] Bozkurt, A., Yaralog̃lu, G. G., Atalar, A., Köymen, H., "A new directional acoustic lens: V-groove lens", in IEEE Ultrasonic Proceedings, 1993.

[5] Briggs, A., Acoustic Microscopy, Oxford, 1992.

[6] Atalar, A., Köymen, H., Degertekin, F., L., "A lamb wave lens for Acoustic Microscopy", IEEE Trans. UFFC, Vol. 39, No. 6, pp. 661-667, 1992.

[7] Kundu, T., Mal, A.K., Weglein, R. D., "Calculation of the acoustic material signature of a layered solid", J. Acous. Soc. Am., type II reflection microscope", IEEE Trans. UFFC, Vol. 34, No.3, pp. 341-345, 1987.

[8] Atalar, A., Köymen, H., "A high efficiency Lamb wave lens for subsurface imaging", Proc. IEEE 1989 Ultrasonics Symposium, pp. 813-816, 1989.

[9] Atalar, A., 'A backscattering formula for acoustic transducers', J. Appl. Phys., Vol.51, pp.3093-3098, 1980.

[10] Auld, B. A., Acoustic Fields and Waves in Solids, John Wiley \& Sons, 1973.

[11] Kino, G. S., Acoustic Waves: Devices, Imaging, and Analog Signal Processing, Prentice-Hall, Inc., 1987. 